\title{
Applying NURBS Surfaces Approximation with Different Parameterization Methods on CKSOM Model Closed Surfaces Data
}

\author{
Seng Poh Lim and Habibollah Haron \\ Soft Computing Research Group, Department of Computer Science, Faculty of Computing, \\ Universiti Teknologi Malaysia, 81310 Skudai, Johor, Malaysia \\ lawrencess87@yahoo.com, habib@utm.my
}

\begin{abstract}
Surface reconstruction towards 3D data is a popular case study in the field of computer graphics. Although many methods are able to solve surface reconstruction problems, but limitations are still appeared. The limitations of Kohonen Self Organizing Map (KSOM) model in closed surface was handled by introducing Cube KSOM (CKSOM) model. However, the CKSOM model output is not in industrial standard format because NURBS are mostly used as surface representation in computer aided geometric design. Furthermore, NURBS surface approximation result will be affected by the parameterization methods. Therefore, the aims are to test and apply NURBS surface approximation on the CKSOM model output and to obtain less surface errors using different parameterization methods. Based on the result, NURBS was proven to be able to apply on the CKSOM model output and uniform parameterization method was proven to be the best method compared to others based on the surfaces error obtained.
\end{abstract}

Keywords: CKSOM model, Closed surfaces data, NURBS, Parameterization, Surface approximation.

\section{Introduction}

Surface reconstruction towards three dimension (3D) data in representing an object surface is very popular in the field of computer graphics. This is due to lot of industries required it in performing some related and important tasks [1]. Although many methods are able to represent the surface and handle the problems of surface reconstruction, but they are still suffer from limitations because the surface represented is not in the industrial standard format for computer graphics. For example, the reconstruction methods are chosen based on the type of data because it can be either in structured or unstructured type [2]. The method selected must also be able to represent the surface after the unstructured data was organized. As shown in the work of [1], the problems of Kohonen Self Organizing Map (KSOM) model and unstructured data as mentioned in [3] were successfully handled by introducing Cube KSOM (CKSOM) model. KSOM model actually was able to represent the surface and solve the unstructured data 
problems for open surfaces, but suffer from limitations in representing and handling the unstructured data problems for closed surfaces. Therefore, CKSOM model is introduced by [1] in handling the limitations. The idea of CKSOM model is merging six two dimension (2D) Kohonen maps to produce a nice closed wireframe surfaces for the talus bones.

Although the connectivity information for each data in forming the object shape has been obtained for CKSOM model, but it still cannot be used as a standard data for the area of computer graphics. This is due to mathematical modeling, such as BSpline and Non Uniform Rational B-Spline (NURBS) are mostly used as surface representation in computer aided geometric design (CAGD) due to their advantages [4]. In addition, there is still no research works which apply NURBS mathematical formulation in forming a closed surfaces shape. For CKSOM model, it forms the closed surface by merging six 2D Kohonen maps. The boundary of each surfaces actually is joined to avoid the holes appeared. Hence, it is a challenging task in representing the closed surface using NURBS mathematical formulation. Furthermore, NURBS surface approximation result will be affected by the parameterization methods as different result will be produced when different parameterization methods were applied. Therefore, the motivations of this paper are intent to test and apply NURBS surface approximation on the CKSOM model closed surface data and to obtain less surface errors using different parameterization methods. NURBS mathematical formula is expected to be able to represent the surface of CKSOM model.

This paper is organized as follows. In Section 2, theories and previous works on NURBS, parameterization and surface approximation are reviewed. Section 3 describes the flows of experiment for NURBS surfaces approximation on CKSOM model closed surfaces data. Analysis and discussion on the experimental results are presented in Section 4. Conclusion and future work are demonstrated in last section.

\section{Literature Review}

\subsection{NURBS Curve and Surfaces}

NURBS is a generalization of Bézier and B-Splines surfaces [2]. NURBS was developed because previous methods suffer from weaknesses. It is widely used in the reverse engineering field and currently is the industrial standards for surface representation [5-7]. NURBS can also be used as approximation method for scattered data and incorporated with most of the current geometric modeling systems [6,8]. The reconstructed surface for NURBS is smoother and it able to deal with non-uniform dataset [9]. Since NURBS is generalized from Bézier and B-Splines, hence related parameters are needed to specify a NURBS curve and surface. Knot vector is used to define the information on how much should be shared by neighbor curves (segments) [10]. Although the data can be in the non-uniform while using NURBS, but it is difficult to handle noisy data. NURBS surface at parameter $(u, v)$ is defined as $S(u, v)$ :

$$
S(u, v)=\sum_{i=1}^{n+1} \sum_{j=1}^{m+1} \frac{P_{i, j} N_{i, k}(u) M_{j, l}(v) h_{i, j}}{N_{i, k}(u) M_{j, l}(v) h_{i, j}}
$$


where $P_{i, j}$ is the three dimensional control net points, $u$ and $v$ are the parameter, $N_{i, k}(u)$ and $M_{j, l}(v)$ are the nonrational B-Spline basis functions, $h_{i, j}$ is weight while $n$ and $m$ is the number of control points. The authors of [11] solved the optimization of NURBS surfaces using linear least squares fitting to approximate the shape. NURBS modeling was used in [12] to solve the shape reconstruction of 3D conducting curved plates. While for [13] work, NURBS is used to detect the curvature, generate the rectangle meshes and also build $U V$ parameter lines. Previous works shows that NURBS is suitable to be applied in the approximation problems.

\subsection{Parameterization and Surface Approximation}

Parameterization and surface approximation are the processes involved in surface reconstruction. Visualization and accuracy of the surface reconstruction result will be affected by the process involved. After the data were reorganized and connectivity of each data was obtained, the parameter for each data on the curve and surface can be obtained through parameterization process. The parameter will be used as the input for B-Spline or NURBS curve and surface to represent the shape of an object through mathematical modeling and simplify complicated 3D problems into 2D tasks [14]. Surface approximation is the process of approximating the generated surface towards original surface using mathematical modeling.

Several parameterization methods along with the characteristic were discussed in the previous works $[6,15,16]$. Uniform method is the simplest method compared to others [16]. While chord length method will be performed using arc length approximation. Centripetal method is the modification based on chord length method [17]. After parameterization was performed, knot vector will be generated using knot vector generation method. Based on [16], equally spaced method is the simplest method to generate knot vector because parameters are not required to perform this method. Parameters from parameterization will be used in averaging knot vector method to generate the knot vector. Based on the parameters and knot vectors generated, hence basis functions and control points for B-Spline or NURBS surfaces can be calculated. Performance of different parameterization methods by using different curve data on B-Spline can be referred from [18] work. The result indicates that the shape and accuracy of curve and surface can be affected by the parameterization methods selected.

\section{Flow of Experiment}

This section discusses the flow of experiment in this paper. The experiment is implemented and images are created using Dev C++ and GNU Plot. Fig. 1 shows the steps followed for NURBS surfaces approximation on CKSOM model closed surfaces data. There are 4 main processes with different number of steps in performing the experiment. Each process with the steps is presented in the following subsections. 


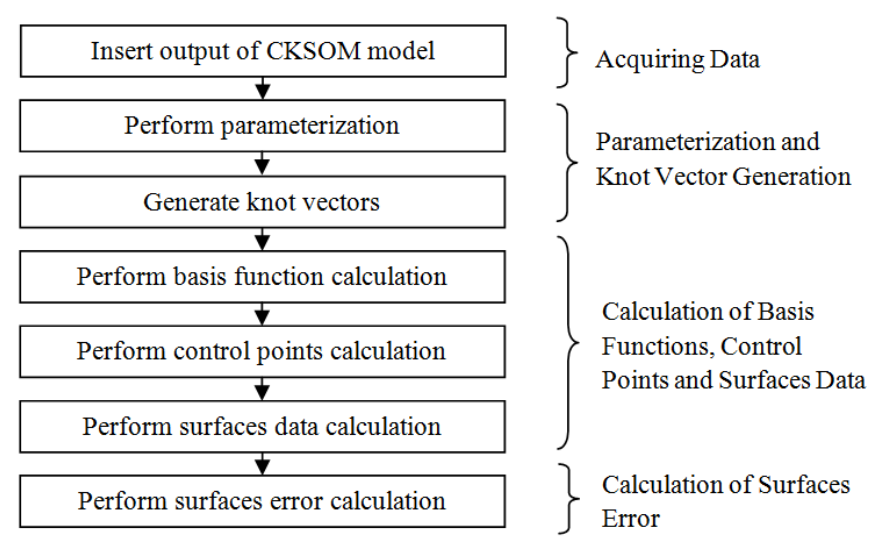

Fig. 1. Flowchart for NURBS surfaces approximation approach

\subsection{Acquiring Data}

The data used for this paper will be the output of CKSOM model, which is the initial sketch of the talus bone (medical image) with the grid size of 20 structured closed surfaces data. They are in 3D coordinates (x, y, z) form. Six 2D Kohonen maps were used to form the CKSOM model in solving the closed surface data problems, hence there will be six surfaces data produced [1]. Each surface data will be inserted and used to perform parameterization and knot vector generation separately in order to obtain control points to generate the NURBS surfaces for talus bone.

\subsection{Parameterization and Knot Vector Generation}

NURBS surfaces approximation approach will be used in this experiment to generate the surfaces data. Perform parameterization and generate knot vectors are the steps involved in this process.

Perform Parameterization. The parameters $(u$ and $v)$ for each surface will be obtained through parameterization process. In order to clearly specify the parameter values of each surface, hence the parameters will be stated in the vector form, $U_{s i}$ and $V_{s j}$, where $U$ is the vector consists of parameter $u$ in horizontal direction and $V$ is the vector consists of parameter $v$ in vertical direction of each surface. While $s$ is the surface number, $i$ is the row and $j$ is the column. As stated in the previous section, CKSOM model used six 2D Kohonen Maps to form the closed surface and they are arranged based on this order (bottom, left, back, right, front, top). Hence, the surface number will be arranged according to this order (bottom $=1$, left $=2$, back $=3$, right $=4$, front $=5$, top $=6$ ). Uniform, centripetal and chord length methods are used to test the approach efficiency. The result will be used to compare the surface errors at the final step. After parameters $\left(U_{s i}\right.$ and $\left.V_{s j}\right)$ for each surface were obtained, in order to 
construct the surface, average parameters of each row and column for each surface will be used to generate the knot vectors. Equations 2 and 3 are referred from [19] will be used to calculate the average parameters for each surface. The equations have been modified by adding the surface number:

$$
\begin{aligned}
s_{s i} & =\frac{u_{s i, 0}+u_{s i, 1}+u_{s i, 2}+\cdots+u_{s i, n}}{n+1} \\
r_{s j} & =\frac{v_{s 0, j}+v_{s 1, j}+v_{s 2, j}+\cdots+v_{s m, j}}{m+1}
\end{aligned}
$$

where $s_{s i}$ are average parameters in the $u$ direction, $r_{s j}$ are average parameters in the $v$ direction, $s$ is the surface number, $i$ is the row and $j$ is the column.

Generate Knot Vectors. Averaging knot vector method is used to generate the knot values for each surface. This method has been suggested and referred from [16] and [20]. Assume that $n+1$ parameter with $u_{0}, u_{1}, \ldots, u_{n}$ and the degree $d$. Therefore, $m+1$ knot will be generated where $m=n+d+1$. Average knot vector is generated as below:

$$
\begin{gathered}
t_{0}=t_{1}=\cdots=t_{d}=0 \\
t_{j+d}=\frac{1}{d} \sum_{i=j}^{j+d-1} u_{j}, j=1,2, \ldots, n-d \\
t_{m-d}=t_{m-d+1}=\cdots=t_{m}=1
\end{gathered}
$$

where $t$ is the knot vector. The equations shown above are applicable for both $U$ and $V$ parameters in order to generate the knot vectors for each surface.

\subsection{Calculation of Basis functions, Control Points and Surfaces Data}

Based on [15], because rational form leads to nonlinear problem, therefore some researchers assigned the weight, $h_{i}=1$ to avoid from the problem. By doing so, the NURBS surface will be reduced to B-Spline surface [16]. According to [21], a curve or surface must used at least with cubic degree (order 4) to represent the generic 3D entities. Therefore, the parameters will be assigned based on the suggestions given. There will be three steps involved in this process.

Perform Basis Function Calculation. Basis function $\left(N_{u}\right.$ and $\left.N_{v}\right)$ for each surface will be calculated based on the generated knot vectors so that control points can be obtained in the next step. Each surface basis function will be separately performed.

Perform Control Points Calculation. Equation 8 will be simplified and used to perform the calculations to determine the control points of NURBS for each surface. 


$$
\begin{aligned}
& N_{u} P N_{v}=D \\
& N_{u}{ }^{T} N_{u} P N_{v} N_{v}{ }^{T}=N_{u}{ }^{T} D N_{v}{ }^{T} \\
& N_{u}{ }^{\prime} P N_{v}{ }^{\prime}=N_{u}{ }^{T} D N_{v}{ }^{T} \\
& N_{u}{ }^{\prime}-1 N_{u}{ }^{\prime} P N_{v}{ }^{\prime} N_{v}{ }^{\prime-1}=N_{u}{ }^{\prime}-1 N_{u}{ }^{T} D N_{v}{ }^{T} N_{v}{ }^{\prime}-1 \\
& P=N_{u}{ }^{\prime}-1 N_{u}{ }^{T} D N_{v}{ }^{T} N_{v}{ }^{\prime}-1
\end{aligned}
$$

where $N_{u}$ and $N_{v}$ is the basis function for each surface, $P$ is the control point, $D$ is the CKSOM model closed surface data, $N_{u}{ }^{T}$ and $N_{v}{ }^{T}$ is the transpose basis function, $N_{u}$, and $N_{v}$ ' is the product of $N_{u}{ }^{T}$ and $N_{u}, N_{v}{ }^{T}$ and $N_{v}, N_{u}{ }^{,-1}$ and $N_{v}{ }^{,-1}$ is the inverse of $N_{u}{ }^{\text {, }}$ and $N_{v}$ '. This kind of concept is used due to different matrix row and column involved in the calculation as the row and column for both basis functions are different.

Perform Surfaces Data Calculation. In order to generate the NURBS surfaces data, equation below will be used:

$$
N_{u} P N_{v}=D^{G}
$$

where $N_{u}$ and $N_{v}$ is the basis function for each surface, $P$ is the control point and $D^{G}$ is the NURBS surface data. After the NURBS surface data for each surface were obtained, comparison on the approximation of NURBS surface data towards CKSOM model closed surface data can be performed in the next process.

\subsection{Calculation of Surfaces Error}

Based on the CKSOM model structure, surfaces error equation is formed based on Euclidean's distance formula:

$$
E=\sum_{s=1}^{6} \sum_{i=1}^{n} \sum_{j=1}^{n}\left|D_{s i j}-D_{s i j}^{G}\right|
$$

where $E$ is total surfaces error between CKSOM model data and NURBS surfaces data, $D_{s i j}$ is the CKSOM model data in $3 \mathrm{D}$ coordinates, $D_{s i j}{ }^{G}$ is the NURBS surfaces data in 3D coordinates, $s$ is the surface number, $n$ is the grid size, $i$ is the row and $j$ is the column in each CKSOM model and NURBS surfaces. Each NURBS surfaces data coordinates $\left(x_{s i j}^{G}, y_{s i j}^{G}, z_{s i j}^{G}\right)$ will be compared with CKSOM model data coordinates $\left(x_{s i j}, y_{s i j}, z_{s i j}\right)$ in order to show the approximation result using surfaces error. In addition, the surfaces error will be compared by using different control points and parameterization methods in order to prove its efficiency. The smaller surfaces error concludes that the more approximate NURBS surfaces data towards CKSOM model data. 


\section{$4 \quad$ Experimental Results}

This section demonstrates the analysis on the experimental results. Number of control points, $C P$ and three parameterization methods were used to test the efficiency of the approaches. Table 1 shows the image results of different control points and parameterization methods. When number of control points increased, better surface was obtained due to more control points being used to adjust the shape of the bone.

Table 1. Image result of different control points and parameterization methods

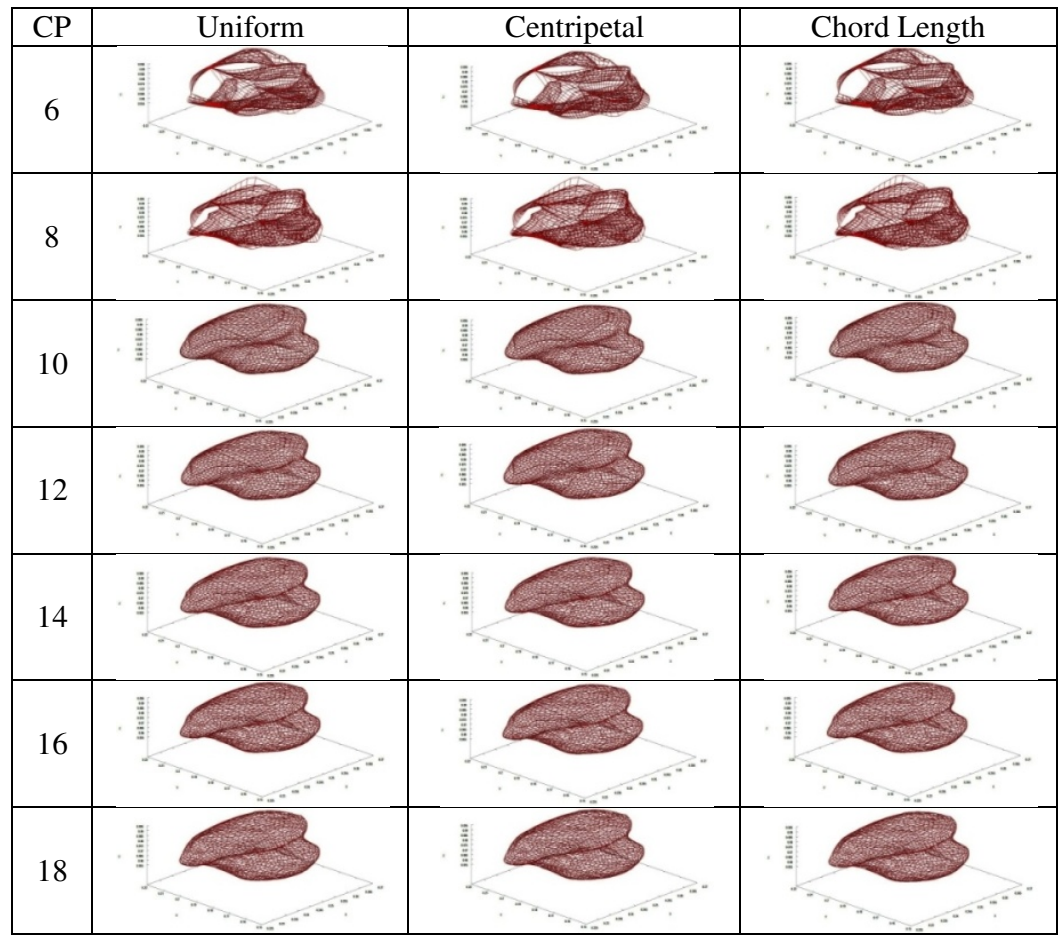

CKSOM model output do not contains holes on the surface because enhancement was performed on KSOM model and handled this problem. However, when CKSOM model output was applied with NURBS, the surface produced contains holes. The holes actually are the linkages between 2 surfaces, which share the same connectivity of data on the edges. Due to different parameters were obtained after parameterization was performed on each surface, therefore different coordinates were obtained on the same location and incorrect surface were produced. The holes size reduced as the number of control points increased. The results also proved that when number of control points is equal to at least half of the grid size, better surface were obtained and the holes are smaller for all parameterization methods. Therefore, the surface of the object is depending on the grid size of CKSOM model and control points of NURBS. The holes appear can be handled by doing some enhancements. 
Based on the result shown in Table 2, surfaces error for various parameterization methods with different control points were decreased as number of control points increased. The results proved that as number of control points increased, the NURBS surfaces produced is more approximate to the CKSOM model output because less surfaces error is obtained. When control points are equal to at least half of the grid size, the surfaces error drop drastically for all parameterization methods. When less control points were used to adjust the shape and the size is less than half of grid size, hence poor result were produced. Due to different parameters values were obtained during parameterization and different coordinates have been generated for the same location of data, hence the surface errors were increased. Therefore, the surface of the object is depending on the grid size of CKSOM model and control points of NURBS.

Table 2. Surfaces error of different control points and parameterization methods

\begin{tabular}{|c|r|r|c|}
\hline $\mathrm{CP}$ & Uniform & Centripetal & $\begin{array}{c}\text { Chord } \\
\text { Length }\end{array}$ \\
\hline 6 & 19.477377 & 19.214752 & 18.927792 \\
\hline 8 & 12.410188 & 12.289616 & 12.172818 \\
\hline 10 & 1.250553 & 1.274489 & 1.312704 \\
\hline 12 & 0.928254 & 0.940571 & 0.963374 \\
\hline
\end{tabular}

\begin{tabular}{|c|c|r|c|}
\hline $\mathrm{CP}$ & Uniform & Centripetal & $\begin{array}{c}\text { Chord } \\
\text { Length }\end{array}$ \\
\hline 14 & 0.680090 & 0.684803 & 0.697408 \\
\hline 16 & 0.461380 & 0.460595 & 0.464579 \\
\hline 18 & 0.269810 & 0.271472 & 0.275149 \\
\hline
\end{tabular}

The parameterization methods do not affect on the surface of the bone because the surface produced for all methods are roughly similar, as shown in the images in Table 1. This concludes that all methods can be used to produce the surface for talus bones. However, the accuracy of the surface errors will be affected by the parameterization methods. Notice that uniform methods performed better in this experiment for approximation problem. It able to obtain less surfaces error compared to centripetal and chord length when number of control points is equal to 10 to 14 and 18 . Chord length method is able to achieve less surfaces error when number of control points is equal to 6 and 8. Centripetal method is able to obtain less surfaces error when number of control points is equal to 16. The result in Table 2 proved that surfaces errors can be affected by parameterization method.

\section{Conclusion and Future Work}

NURBS surface approximation was proven to be able to apply on the output of CKSOM model. However, based on the image results, the surface of the bone contains holes when NURBS was applied. This is due to different surface parameters were produced based on the approach, hence incorrect range of knot vectors as boundary will be shared by the same location of data. This is because same location data have different coordinates. As a result, surface errors were increased and incorrect shape which contains holes was obtained. For the data from the same location, they should have the same parameters. Uniform parameterization method was proven to be the best method compared to others based on the surface errors obtained.

Future work should consider in enhancing and improving theory. The holes on the surface should be solved by doing enhancement. Although the data represent different 
surface, since it is the same location, hence the data must only contain one parameter value. Therefore, parameters and control points for each surface should be standardized in the beginning before the surface data were generated. In addition, optimization can be performed by reducing the surfaces error. Mathematical or soft computing methods, such as Genetic Algorithm, Differential Evolution or Particle Swarm Optimization can be applied to solve the issues. As stated by [22] and [23], performance of soft computing field is a good research field to be further explored. Hence the optimization on surfaces error is suitable to be applied using soft computing methods.

Acknowledgement. This work is financed by UTM Zamalah.

\section{References}

1. Lim, S.P., Haron, H.: Cube Kohonen Self-Organizing Map (CKSOM) Model With New Equations in Organizing Unstructured Data. IEEE Transactions on Neural Networks and Learning Systems 24(9), 1414-1424 (2013)

2. Lim, S.P., Haron, H.: Surface Reconstruction Techniques: A Review. Artif Intell Rev., 1-20 (2012), doi:10.1007/s10462-012-9329-z

3. Lim, S.P., Haron, H.: Applying Kohonen Network in Organising Unstructured Data for Talus Bones. In: ICTMF 2012, Lecture Notes in Information Technology, vol. 38, pp. 378-384 (2012)

4. Adi, D.I.S., Shamsuddin, S.M., Ali, A.: Particle Swarm Optimization for NURBS Curve Fitting. In: Sixth International Conference on Computer Graphics, Imaging and Visualization, pp. 259-263 (2009)

5. Tsai, Y.C., Huang, C.Y., Lin, K.Y., Lai, J.Y., Ueng, W.Y.: Development of Automatic Surface Reconstruction Technique in Reverse Engineering. Int. J. Adc. Manuf. Technol. 42(1-2), 152-167 (2008)

6. Rogers, D.F.: An Introduction to NURBS With Historical Perspective. Morgan Kaufmann Publishers (2001)

7. He, Y., Qin, H.: Surface Reconstruction with Triangular B-splines. In: Proceedings of the Geometric Modeling and Processing 2004, pp. 279-287 (2004)

8. Hoffmann, M.: Numerical Control Of Kohonen Neural Network For Scattered Data Approximation. Numerical Algorithm 39, 175-186 (2005)

9. Zhao, H.K., Osher, S., Fedkiw, R.: Fast Surface Reconstruction Using the Level Set Method. In: IEEE Workshop on Variational and Level Set Methods, pp. 194-201 (2001)

10. Miléŕ, V., Miléŕ, J.: NURBS Curves and Surfaces (2005), http: / / www. rw-designer. com/NURBS (retrieved)

11. Goldenthal, R., Bercovier, M.: Design of Curves and Surfaces Using Multi-Objective Optimization (2004), http: / / leibniz.cs.huji.ac.il/tr/741.pdf (retrieved)

12. Saeedfar, A., Barkeshli, K.: Shape Reconstruction of Three-Dimensional Conducting Curved Plates Using Physical Optics, NURBS Modeling, and Genetic Algorithm. IEEE Transactions on Antennas And Propagation 54(9), 2497-2507 (2006)

13. Meng, F., Wu, L., Luo, L.: 3D Point Clouds Processing and Precise Surface Reconstruction of the Face. In: 2010 International Conference on Image Analysis and Signal Processing, pp. 104-107 (2010) 
14. Cartade, C., Mercat, C., Malgouyres, R., Samir, C.: Mesh Parameterization with Generalized Discrete Conformal Maps. Journal of Mathematical Imaging and Vision 46(1), 1-11 (2013)

15. Piegl, L., Tiller, W.: The NURBS Book, 2nd edn. Springer-Verlag New York, Inc. (1997)

16. Adi, D.I.S., Shamsuddin, S.M., Hashim, S.Z.M.: NURBS Curve Approximation using Particle Swarm Optimization. In: Seventh International Conference on Computer Graphics, Imaging and Visualization, pp. 73-79 (2010)

17. Lee, E.T.: Choosing Nodes in Parametric Curve Interpolation. Computer Aided Design 21, 363-370 (1989)

18. Haron, H., Rehman, A., Adi, D.I.S., Lim, S.P., Saba, T.: Parameterization Method on B-Spline Curve. Mathematical Problems in Engineering 2012, 1-22, Article ID 640472 (2011), doi:10.1155/2012/640472

19. Shene, C.K.: Parameters and Knot Vectors for Surfaces (2005), http: / / www.cs.mtu. edu/shene/COURSES/cs3621/NOTES / INT-APP/PARA-surface.html (retrieved)

20. Forkan, F., Shamsuddin, S.M.: Kohonen-Swarm Algorithm for Unstructured Data in Surface Reconstruction. In: Fifth International Conference on Computer Graphics, Imaging and Visualization, pp. 5-11 (2008)

21. Kumar, G.S., Kalra, P.K., Dhande, S.G.: Parameter Optimization for B-spline Curve Fitting using Genetic Algorithms. In: The 2003 Congress on Evolutionary Computation, pp. 1871-1878 (2003)

22. Lim, S.P., Haron, H.: Performance of Different Techniques Applied in Genetic Algorithm towards Benchmark Functions. In: Selamat, A., Nguyen, N.T., Haron, H. (eds.) ACIIDS 2013, Part I. LNCS, vol. 7802, pp. 255-264. Springer, Heidelberg (2013)

23. Lim, S.P., Haron, H.: Performance Comparison of Genetic Algorithm. In: Differential Evolution and Particle Swarm Optimization Towards Benchmark Functions, ICOS 2013, pp. 41-46. IEEE Computer Chapter (2013) 